\title{
Diabetes-associated genetic variation in TCF7L2 alters pulsatile insulin secretion in humans
}

\author{
Marcello C. Laurenti, ${ }^{1}$ Chiara Dalla Man, ${ }^{2}$ Ron T. Varghese, ${ }^{1}$ James C. Andrews, ${ }^{3}$ Robert A. Rizza, ${ }^{1}$ \\ Aleksey Matveyenko, ${ }^{1,4}$ Giuseppe De Nicolao, ${ }^{5}$ Claudio Cobelli, ${ }^{2}$ and Adrian Vella ${ }^{1}$ \\ 'Division of Endocrinology, Diabetes \& Metabolism, Mayo Clinic, Rochester, Minnesota, USA. ${ }^{2}$ Department of Information \\ Engineering, University of Padova, Padova, Italy. ${ }^{3}$ Vascular and Interventional Radiology and ${ }^{4}$ Physiology and Biomedical \\ Engineering, Mayo Clinic, Rochester, Minnesota, USA. 'Department of Computer Engineering and Systems Science, \\ University of Pavia, Pavia, Italy.
}

BACKGROUND. Metabolic disorders such as type 2 diabetes have been associated with a decrease in insulin pulse frequency and amplitude. We hypothesized that the T allele at rs7903146 in TCF7L2, previously associated with $\beta$ cell dysfunction, would be associated with changes in these insulin pulse characteristics.

METHODS. Twenty-nine nondiabetic subjects (age $46 \pm 2$, BMI $28 \pm 1 \mathrm{~kg} / \mathrm{m}^{2}$ ) participated in this study. Of these, 16 were homozygous for the $C$ allele at rs7903146 and 13 were homozygous for the T allele. Deconvolution of peripheral C-peptide concentrations allowed the reconstruction of portal insulin secretion over time. These data were used for subsequent analyses. Pulse orderliness was assessed by approximate entropy (ApEn), and the dispersion of insulin pulses was measured by a frequency dispersion index (FDI) after a Fast Fourier Transform (FFT) of individual insulin secretion rates.

RESULTS. During fasting conditions, the CC genotype group exhibited decreased pulse disorderliness compared with the TT genotype group ( $1.10 \pm 0.03$ vs. $1.19 \pm 0.04, P=0.03$ ). FDI decreased in response to hyperglycemia in the CC genotype group, perhaps reflecting less entrainment of insulin secretion during fasting.

CONCLUSION. Diabetes-associated variation in TCF7L2 is associated with decreased orderliness and pulse dispersion, unchanged by hyperglycemia. Quantification of ApEn and FDI could represent novel markers of $\beta$ cell health.

FUNDING. This work was funded by US NIH (DK78646, DK116231), University of Padova research grant CPDA145405, and Mayo Clinic General Clinical Research Center (UL1 TR000135).

Conflict of interest: The authors have declared that no conflict of interest exists.

Copyright: () 2020, American Society for Clinical Investigation.

Submitted: January 3, 2020

Accepted: March 5, 2020

Published: April 9, 2020

Reference information: /CI Insight. 2020;5(7):e136136.

https://doi.org/10.1172/jici.

insight.136136.

\section{Introduction}

Type 2 diabetes is a common, but complex, metabolic disorder characterized by defects in islet function during the fasting and postprandial states that result in hyperglycemia. The disorder arises out of a complex interaction between genetic predisposition and environmental exposures (chiefly those affecting nutritional intake and energy expenditure). To date, genome-wide association studies have identified more than 200 loci harboring common genetic variants that predispose to type 2 diabetes (1), although the effect size of many is small (1-3). Most of these variants influence $\beta$ cell function and insulin secretion (4). Of these common variants, the greatest predisposition to disease is arguably conferred by the $\mathrm{T}$ allele at rs7903146 in TCF7L2 $(1,3)$, which is associated with a subtle defect in insulin secretion (5). This results in impaired compensation for a decrease in insulin action, as evident by comparison of the hyperbolic relationship between $\beta$ cell responsivity and insulin action in subjects with and without 2 copies of the diabetes-associated (T) allele (6). Impaired postprandial suppression of glucagon secretion (6) is also likely to contribute to postprandial hyperglycemia, at least in situations where insulin secretion is impaired (7). 
The secretion of insulin by $\beta$ cells is pulsatile and arises from a complex interplay between cellular metabolism and electrophysiology (8). Although individual $\beta$ cells have a pulse periodicity of approximately 5 minutes, the pulses of insulin secretion seen in the portal circulation represent the summation of multiple asynchronous clusters of $\beta$ cells entrained to secrete synchronously by metabolic $(9,10)$ or other stimuli $(11,12)$. After insulin is secreted into the portal circulation, it undergoes hepatic extraction (13) so that, by the time they appear in the systemic circulation, insulin pulses are attenuated and difficult to discern (14). The degree of hepatic extraction of portal insulin seems to be influenced by the amount of insulin secreted, whether in response to an oral challenge (15) or the size of the pulse mass (16). Therefore, accurate identification of insulin pulses needs to account for hepatic insulin extraction.

The study of insulin pulse characteristics in humans, to date, has suggested that, in people with type 2 diabetes, both pulse amplitude and pulse frequency are decreased compared with healthy subjects (17). Indeed, defective insulin pulsatility has been observed in first-degree relatives of people with diabetes prior to the development of overt hyperglycemia (18). Similar defects have been associated with obesity and with aging (19). Insulin secretagogues can increase pulse amplitude in people with type 2 diabetes. However, pulse frequency is unchanged, suggesting that decreased pulse periodicity is a feature of diseased islets (20, 21). Given the complexity of the events leading to insulin synthesis and packaging (22), as well as pulse generation in the $\beta$ cell (8), it is possible that abnormal pulse characteristics may serve as an early marker of $\beta$ cell dysfunction that precedes the development of hyperglycemia, especially in people genetically predisposed to type 2 diabetes by family history (18) or - more specifically - due to genetic variation associated with impaired insulin secretion, as is the case for the T allele at rs7903146 $(6,23)$. We therefore sought to determine whether nondiabetic subjects homozygous for the diabetes-associated allele at rs7903146 (TT genotype) exhibited detectable abnormalities in insulin pulse secretion.

To do so, we developed a potentially novel method that utilizes nonparametric stochastic deconvolution applied to peripheral C-peptide concentrations after determination of C-peptide clearance in each subject $(24,25)$. The reconstructed insulin secretion, used for this analysis, demonstrated pulse characteristics comparable with those obtained from hepatic vein insulin concentrations, while avoiding the confounding effects of hepatic extraction (24).

Although the basal and above-basal components of the data series did not exhibit significant differences between genotype groups, we observed increased disorderliness of insulin pulses (26) in subjects with diabetes-associated variation at rs7903146. These differences were only present during the fasting phase of the experiment.

To better understand the underlying frequencies and amplitudes of the pulse series that, together, produce the minute-to-minute variation in insulin secretion (18, 27-29), we used a Fast Fourier Transform (FFT) of the insulin secretion rate over the duration of the 2 phases of the experiment. In the CC - but not in the TT - genotype group, we noted a decrease in the dispersion of pulse frequencies in response to hyperglycemia, as measured by a frequency dispersion index (FDI) (30). In contrast, FDI in the TT group was relatively unchanged by hyperglycemia. These data suggest that diabetes-associated variation in TCF7L2 is associated with decreased pulse orderliness before overt defects in $\beta$ cell function are apparent. Pulse disorderliness and ( $n$ unchanging) dispersion of pulse frequencies during fasting may be a novel marker of impaired $\beta$ cell health.

\section{Results}

Volunteer characteristics. Subject characteristics grouped by genotype at rs7903146 are summarized in Table 1. There were no significant anthropometric differences between genotype groups. Minimal model indices of insulin secretion and action also did not differ between groups. Note that, in 1 subject, due to loss of venous access, we only have data from the fasting phase of the experiment (Supplemental Figures 1-6; supplemental material available online with this article; https://doi.org/10.1172/jci.insight.136136DS1).

Plasma glucose, insulin, C-peptide concentrations, and insulin secretion rate during the experiment. Mean glucose (Figure 1A) during the fasting phase of the experiment did not differ between groups. Although mean glucose during the hyperglycemic clamp was slightly and significantly increased (Table 1) in the TT group, these differences were not sustained.

Hepatic vein insulin concentrations (Figure 1B) and C-peptide concentrations (Figure 1C) did not differ during either phase of the experiment (Table 1). Nonparametric deconvolution of peripheral C-peptide concentrations allowed the reconstruction of individualized insulin secretion rates used for this analysis. 
Table 1. Subject characteristics by genotype group.

\begin{tabular}{|c|c|c|c|}
\hline & CC & TT & $P$ value \\
\hline$n(M / F)$ & $16(5 / 11)$ & $13(5 / 8)$ & $0.29^{A}$ \\
\hline Age (years) & $45 \pm 3$ & $47 \pm 3$ & 0.57 \\
\hline Lean body mass (Kg) & $47.4 \pm 2.1$ & $49.1 \pm 3.9$ & 0.69 \\
\hline \multirow[t]{2}{*}{ BMI $\left(\mathrm{kg} / \mathrm{m}^{2}\right)$} & $28.0 \pm 1.1$ & $26.8 \pm 0.7$ & 0.38 \\
\hline & \multicolumn{2}{|c|}{ Screening OGTT data } & \\
\hline$n$ (NGT/IGT) & $10 / 6$ & $7 / 6$ & $0.27^{\mathrm{A}}$ \\
\hline$S_{i}\left(1 \times 10^{-4} \mathrm{dL} / \mathrm{kg} / \mathrm{min}\right.$ per $\left.\mu \mathrm{U} / \mathrm{mL}\right)$ & $15 \pm 3$ & $15 \pm 3$ & 0.95 \\
\hline$\phi_{\text {dynamic }}\left(1 \times 10^{-9}\right)$ & $700 \pm 70$ & $605 \pm 77$ & 0.29 \\
\hline$\phi_{\text {static }}\left(1 \times 10^{-9} \mathrm{~min}^{-1}\right)$ & $48 \pm 3$ & $44 \pm 4$ & 0.27 \\
\hline$\phi\left(1 \times 10^{-9} \mathrm{~min}^{-1}\right)$ & $55 \pm 3$ & $51 \pm 4$ & 0.41 \\
\hline Mean glucose during hyperglycemia (mmol/L) & $8.6 \pm 0.1$ & $8.9 \pm 0.2$ & 0.05 \\
\hline Mean insulin during fasting (pmol/L) & $19 \pm 3$ & $14 \pm 1$ & 0.10 \\
\hline Mean insulin during hyperglycemia (pmol/L) & $72 \pm 8$ & $64 \pm 8$ & 0.47 \\
\hline Mean C-peptide during fasting (nmol/L) & $0.59 \pm 0.04$ & $0.51 \pm 0.04$ & 0.27 \\
\hline Mean C-peptide during hyperglycemia (nmol/L) & $1.48 \pm 0.10$ & $1.36 \pm 0.12$ & 0.46 \\
\hline
\end{tabular}

BMI, body mass index; NGT, normal glucose tolerance; ICT, impaired glucose tolerance; $\Phi, \beta$ cell responsivity; $S_{i}$ insulin action; OCTT, oral glucose tolerance test; $\mathrm{DI}$, disposition index. Values reported are means $\pm \mathrm{SEM}$. $P$ value reports the result of an unpaired, 2-tailed $t$ test or a 2-tailed Wilcoxon test for values that are not normally distributed. ${ }^{A} P$ value is the result of a $\chi^{2}$ test.

The mean rate of insulin secretion did not differ during either phase of the experiment for the 2 groups (Figure 1D).

Insulin secretion rate from representative subjects and individual basal insulin secretion rate, and insulin pulse amplitude during fasting and during hyperglycemia, in each genotype group. Representative individual insulin secretion rates (obtained by deconvolution of peripheral C-peptide concentrations) of those analyzed are shown during the fasting (Figure 2A) and hyperglycemic (Figure 2C) phases of the experiment. Basal, above-basal, and pulse amplitude were calculated for each individual.

The nonpulsatile component (basal) of insulin secretion (Figure 2B) did not differ during fasting (142 \pm 13 vs. $132 \pm 13 \mathrm{pmol} / \mathrm{min}$; CC vs. TT, respectively; $P=0.71)$. Basal secretion rose significantly in response to hyperglycemia but did not differ between groups (394 $\pm 36 \mathrm{vs.} 411 \pm 35 \mathrm{pmol} / \mathrm{min}, P=0.68$ ).

Insulin pulse amplitude (Figure 2D) did not differ during fasting (59 \pm 8 vs. $60 \pm 6 \mathrm{pmol} / \mathrm{min}, P$ $=0.88$ ). Pulse amplitude also rose significantly in response to hyperglycemia but did not differ between groups (164 \pm 21 vs. $185 \pm 20 \mathrm{pmol} / \mathrm{min}, P=0.27$ ).

Individual ApEn during fasting and during hyperglycemia in each genotype group. Pulse orderliness as measured by approximate entropy (ApEn) differed significantly during fasting (1.10 \pm 0.03 vs. $1.19 \pm$ $0.04, P=0.03$ ), with a higher score (increased disorderliness) in the TT group (Figure $3 \mathrm{~A})$. No differences in ApEn were apparent during hyperglycemia (Figure 3B; $1.18 \pm 0.03$ vs. $1.17 \pm 0.03 ; P=0.92$ ).

Periodogram analysis, individual pulse interval, and FDI during fasting and during hyperglycemia in each genotype group. The Fourier power spectra shown are representative of those obtained during fasting (Figure 4A) and during hyperglycemia (Figure 4C); they were then used to calculate pulse interval and FDI (Supplemental Figures 3-6).

Subjects with the CC genotype showed a nonsignificant trend toward slower pulse intervals during fasting (Figure $4 \mathrm{~B} ; 6.2 \pm 0.6 \mathrm{~min}$ vs. $4.9 \pm 0.3 \mathrm{~min} ; P=0.06$ ). In response to hyperglycemia, pulse interval did not change significantly (CC, $P=0.09$; TT, $P=0.41$ ) within genotype groups.

The FDI (Figure 4D) did not differ significantly between genotype groups during fasting. However, in response to hyperglycemia, the dispersion of pulse frequencies decreased significantly in the CC group 

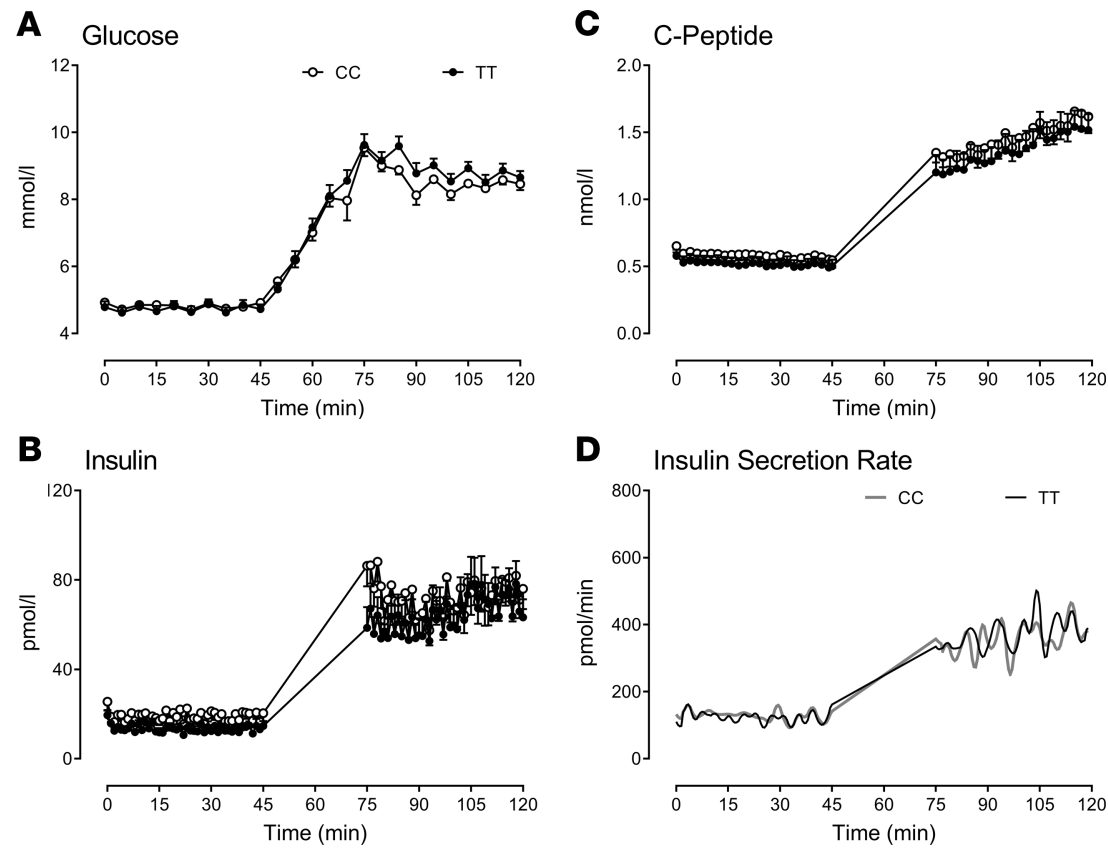

Figure 1. Hormone and substrate concentrations during the experiment. Glucose (A), insulin (B), C-peptide (C), and insulin secretion rate (D) during the experiment in subjects with the CC (open circles) and TT (solid circles) genotypes. Values plotted are means \pm SEM.

$(0.48 \pm 0.02$ vs. $0.41 \pm 0.01, P=0.02)$ but not in the TT group $(P=0.41)$. The between-group change in FDI (fasting vs. hyperglycemia) was not significant $(P=0.25)$

Pulse characteristics attributable to glucose tolerance status. To ensure that differences in pulse characteristics could not be explained by increased numbers of impaired glucose tolerance (IGT) in one genotype group versus the other, we compared pulse characteristics in those with normal glucose tolerance (NGT) versus those with IGT (Table 2). Despite the presence of impaired $\beta$ cell function in response to a $75 \mathrm{~g}$ oral glucose tolerance test (OGTT) at the time of screening, no significant differences in pulse characteristics were associated with glucose tolerance status.

\section{Discussion}

This is the first application of our recently described methodology that utilizes peripheral C-peptide concentrations to deconvolve pulsatile insulin secretion into the portal vein without the potential confounding effects of hepatic extraction (24). The ability to measure high-frequency pulsatile insulin secretion without hepatic vein catheterization raises the possibility of performing larger studies across the spectrum of glucose tolerance to follow up and to replicate the findings of the present study. Diabetes-associated variation in TCF7L2 is associated with a mild impairment in insulin secretion for a given degree of insulin action (6). However, in this small sample size, when subjects were matched for age, sex, weight, and fasting glucose, we likely lacked the statistical power to discern differences in insulin secretion and action in response to a $75 \mathrm{~g}$ OGTT. Nevertheless, pulse analysis revealed that the diabetes-associated allele in TCF7L2 was associated with a decrease in pulse orderliness, as measured by ApEn (31). This could not be explained by changes in $\beta$ cell responsivity and insulin action measured during the OGTT, as evidenced by a lack of correlation with these indices (data not shown), and the absence of similar differences between people with NGT and IGT (Table 2).

What is the mechanism underlying these changes? Multiple, well-powered, in vivo studies including ours - measuring $\beta$ cell function in response to an oral challenge demonstrate an association of the diabetes-associated allele (T) at rs7903146 with impaired insulin secretion $(6,23,32-34)$. While it is possible that diabetes-associated variation in TCF7L2 is not the etiologic variant but is in linkage disequilibrium with an etiologic variant in another locus, or controls another locus (35), this seems unlikely (36), since multiple experiments implicate TCF7L2 in the pathogenesis of type 2 diabetes. 

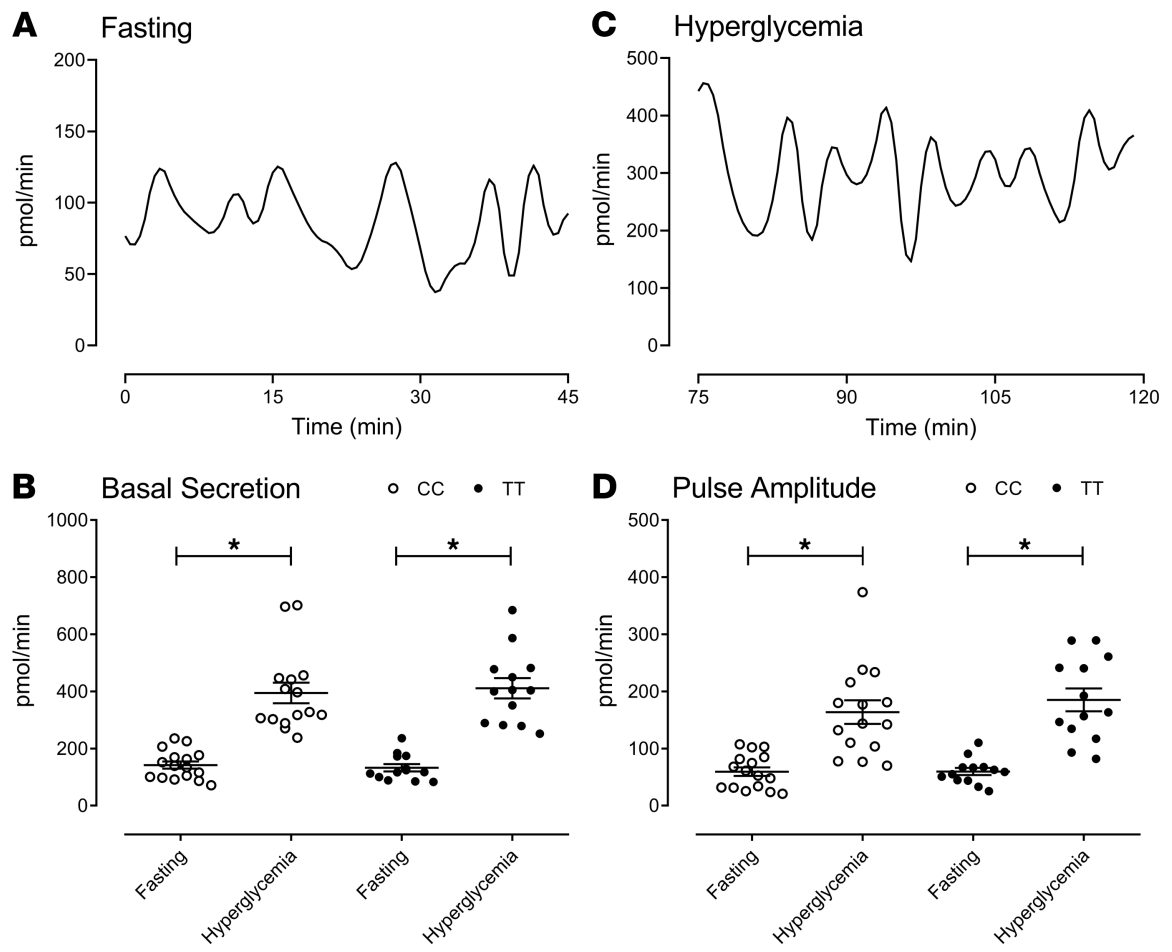

Figure 2. Insulin secretion rates used to derive basal and pulsatile secretion. Insulin secretion rate in a representative individual during the fasting (A) and hyperglycemic phase of the experiment (C). Individual basal (B) and amplitude (D) of insulin peaks in the fasting and hyperglycemic phase of the experiment are also shown for the CC (open circles) and TT (solid circles) genotypes. Also shown are means \pm SEM for each group. ${ }^{*} P<0.05$ using a Wilcoxon matched-pairs signed rank test to examine within-group changes (fasting vs. hyperglycemia).

For example, decreasing TCF7L2 expression in mature mouse islets impaired glucose-stimulated insulin secretion (37). Intriguingly, a subsequent study in which TCF7L2 expression was decreased demonstrated normal secretion in response to $\mathrm{K}^{+}$- or $\mathrm{Ca}^{2+}$-induced depolarization, suggesting a defect in glucose sensing (38). No decrease in insulin granules was observed, although the subcellular distribution was changed in a manner suggesting that TCF7L2 knockdown altered granule recruitment and tethering to the cell membrane (38). Similar results were observed in islets from humans heterozygous or homozygous for the diabetes-associated allele at rs7903146 (39).

In contrast, TCF7L2 KO resulted in decreased islet mass and islet coordination, as well as a corresponding decrease in $\beta$ cell responsiveness to glucose (40). TCF7L2 activates $\beta$-catenin. Overexpression of TCF7L2, as observed in type 2 diabetes (33), sequesters $\beta$-catenin in the nucleus, disrupting the actin cytoskeleton and insulin vesicle trafficking (41). While diabetes-associated genetic variation in TCF $2 L 2$ is unlikely to have dramatic effects on $\beta$ cell function comparable with those observed with gene silencing or overexpression experiments, the experimental data support a role of TCF7L2 in coordinating insulin vesicle trafficking and docking with the cell membrane prior to release.

Another surprising finding is that the diabetes-protective allele showed a tendency to have longer pulse intervals during fasting compared with the pulse intervals observed in those individuals homozygous for the $\mathrm{T}$ allele at rs 7903146. Decreased pulse frequency has been thought to represent impairment in $\beta$ cell health $(17,42,43)$; however, in this case — while not statistically significant — during the fasting state, the opposite was observed; people with diabetes-protective variation in the TCF7L2 locus had a tendency to a longer main pulse interval. On the other hand, a mean measurement of time interval between pulses may not fully characterize the contribution of different islets' secretion rates and pulse frequencies to net insulin secretion. We therefore applied a FDI in order to estimate the dispersion of insulin frequencies contributing to insulin secretion in a given individual. With this in mind, we measured frequency dispersion. It is notable that FDI is positively correlated with lengthening pulse interval in the fasting state, increasing as the average pulse interval lengthens (Supplemental Figure 7). We also observed that pulse frequency dispersion decreased in response to hyperglycemia in 

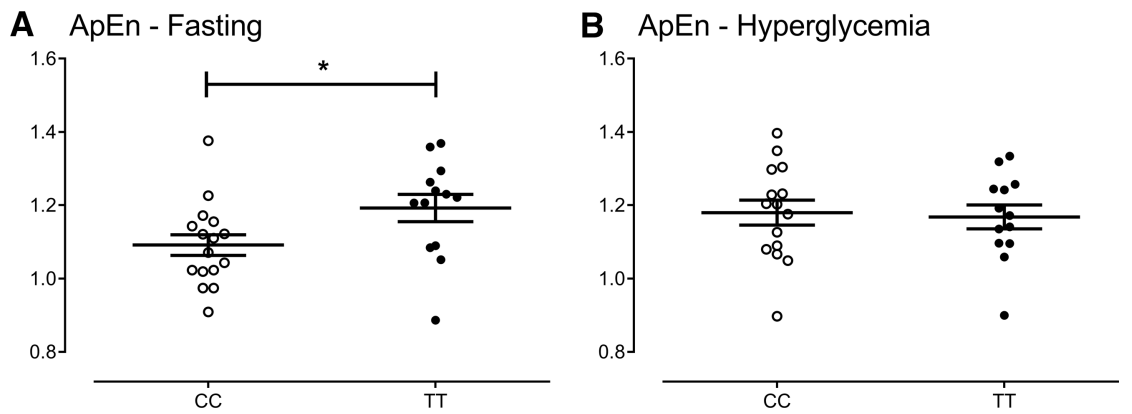

Figure 3. Pulse orderliness measured by ApEn. Individual ApEn in the fasting (A) and hyperglycemic phases of the experiment (B) are shown for the CC (open circles) and TT (solid circles) genotypes. Also shown are means \pm SEM for each group. ${ }^{*} P<0.05$ using a 2 -tailed Wilcoxon test.

the CC, but not in the TT, genotype group. This suggests that, in health, if multiple, small asynchronous pulses contribute to insulin secretion, the main contributions to insulin secretion can occur at a lower frequency.

During the hyperglycemic clamp, there was a slight but significant tendency to higher glucose concentrations in the TT group. While this may have masked between-group differences in insulin pulsatility in response to glucose elevation, this cannot explain the differences present in the fasting phase of the experiment. Also, net insulin secretion increased similarly between groups, while pulse characteristics (other than amplitude) were essentially unchanged in the TT group in response to hyperglycemia. Future studies manipulating insulin action (6) or fasting insulin secretion (43) will be required to determine if the relatively fixed FDI in the group with diabetes-associated variation in TCF7L2 reflects a necessity to entrain more islets in the basal fasting state to maintain euglycemia.

It is also important to note that this study was powered to detect the effect of diabetes-associated genetic variation in TCF7L2 - focusing on the common variant (rs7903146) with the greatest effect on disease predisposition. To maximize our power to detect an effect, we recruited by genotype, selecting subjects homozygous for the disease-associated (TT) and the disease-protective (CC) variants. Unfortunately, this sample size and recruitment method means that we will have insufficient power to reliably detect an effect of other common variants with lesser effect sizes on insulin pulse characteristics (3). Fortunately, our methodology should allow the development of larger cohorts where these questions can be addressed.

Prior experiments using the oral minimal model to measure $\beta$ cell function (44) have suggested that, while $\beta$ cell responsivity to glucose is relatively unchanged across the spectrum of glucose tolerance, it is inappropriate for the degree of insulin action that is present as glucose tolerance worsens $(45,46)$. Despite the inclusion of people with some degree of glucose intolerance, none of the between-group differences in pulse characteristics could be explained by their glucose tolerance status. Pulse characteristics also did not correlate with the standard indices of insulin secretion and action obtained from the minimal model. This may be, in part, due to the experimental design where efforts were made to ensure that fasting glucose concentrations at the time of screening did not differ between genotype groups in the fasting state. Similarly, a hyperglycemic clamp was utilized to ensure that glucose concentrations did not differ between groups during hyperglycemia. None of the subjects studied had impaired fasting glucose; therefore, future experiments will be required to determine if people with impaired fasting glucose, with or without IGT, have altered insulin pulse characteristics compared with people with normal fasting glucose.

In conclusion, people predisposed to type 2 diabetes because of diabetes-associated genetic variation at rs7903146 exhibit subtle differences in insulin pulse characteristics compared with those without risk alleles. Increased pulse disorderliness occurred independently of changes in more conventional measures of $\beta$ cell function. This raises the possibility that pulse characteristics of $\beta$ cell insulin secretion may serve as additional, independent measures of $\beta$ cell health. Since this will require additional experiments to determine their significance and utility, the use of our methodology - which eschews the need of a hepatic vein catheter - is an advantage. Future studies in larger numbers of subjects are now feasible and may help validate these potentially novel measures of $\beta$ cell function and dysfunction. 

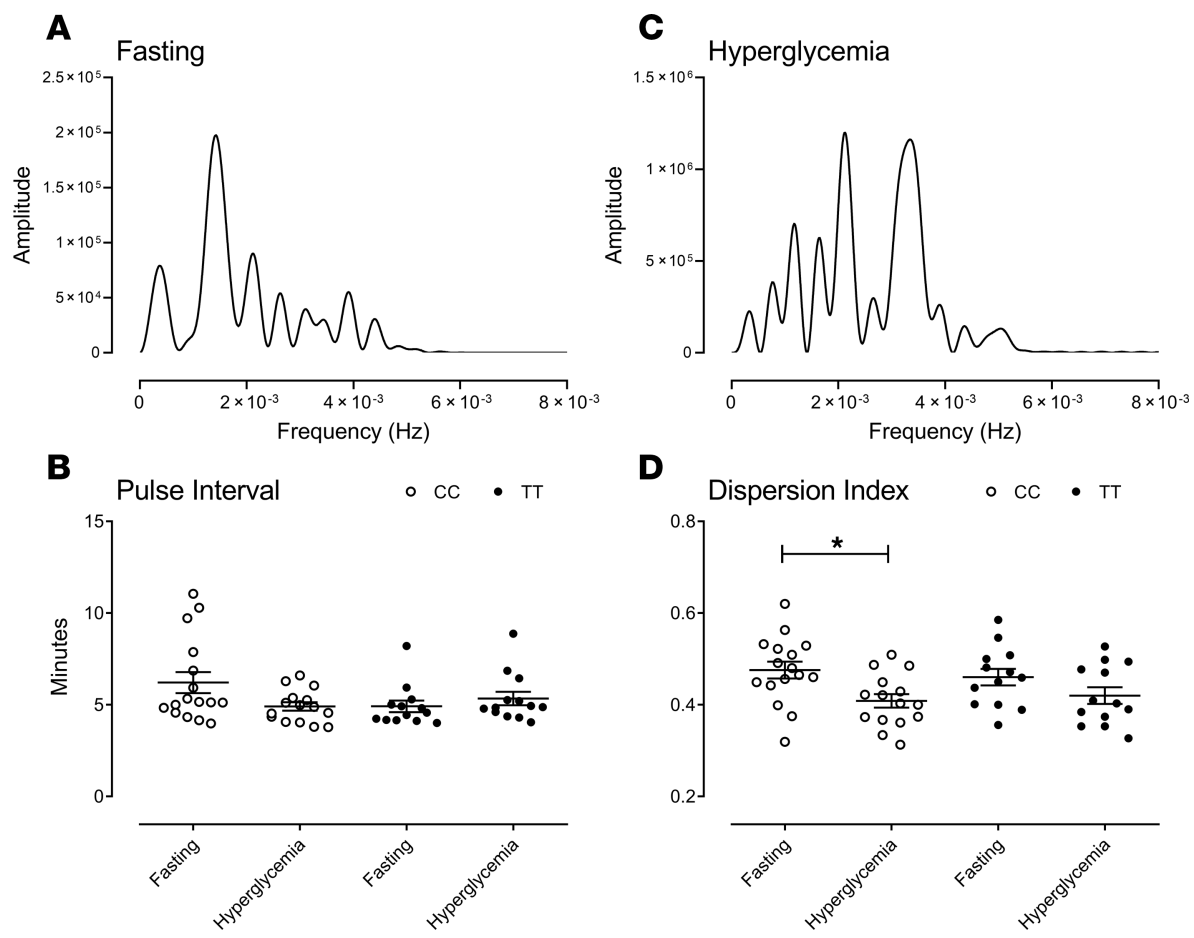

Figure 4. Periodograms used to measure pulse interval and frequency dispersion. Periodograms in a representative individual during the fasting (A) and hyperglycemic phase of the experiment (C). Individual pulse interval (B) and frequency dispersion index (D) in the fasting and hyperglycemic phase of the experiment are also shown for the CC (open circles) and TT (solid circles) genotypes. Also shown are means \pm SEM for each group. ${ }^{*} P<0.05$ using a Wilcoxon matched-pairs signed rank test to examine within-group changes (fasting vs. hyperglycemia).

\section{Methods}

Study subjects. The subject (10 males and 19 females) characteristics are described in Table 1 . They underwent a screening exam to ensure that they were healthy and had no active illness, had no prior history of diabetes mellitus, and were not taking medications that might alter glucose metabolism. A 2-hour, 7-sample OGTT was used at the time of screening to measure insulin secretion and action as before (47). Body composition was measured by dual energy X-ray absorptiometry (iDXA scanner; GE Healthcare). Participants met with a nutritionist and followed a weight maintenance diet (55\% carbohydrate, $30 \%$ fat, and $15 \%$ protein) for a minimum of 3 days prior to the study visit.

Experimental design. As previously outlined in ref. 24, subjects were admitted to the Clinical Research and Translation Unit (CRTU) at 6 p.m. on the day prior to study. They then consumed a standard meal and fasted overnight. The following morning (at approximately 6:30 a.m.), an $18 \mathrm{~g}$ cannula was inserted retrogradely into a dorsal hand vein. This was then placed in a heated Plexiglas box maintained at $55^{\circ} \mathrm{C}$ to allow sampling of arterialized venous blood. Subjects were then moved to the radiology suite, where a hepatic vein catheter was placed via the femoral vein under fluoroscopic guidance $(16,48)$. Following their return, at 8 a.m. ( $0 \mathrm{~min})$, blood was sampled at 1 -minute intervals from the arterialized hand vein and from the hepatic vein at 2-minute intervals over a 45-minute period (Fasting Phase). At 8:46 a.m. (46 min), glucose infusion commenced, and the infusion rate was adjusted to rapidly achieve and maintain peripheral glucose concentrations of approximately $9.8 \mathrm{mmol} / \mathrm{L}$. Following this, 30-minute interval (9:15 a.m.) blood was sampled during the Hyperglycemic Phase for an additional 45 minutes. At 10 a.m. (120 min), all cannulas and the hepatic vein catheter were removed. Participants consumed a meal and left the CRTU.

Analytic techniques. Plasma samples were placed on ice, centrifuged at $1750 \mathrm{~g}$ for 15 minutes at $4^{\circ} \mathrm{C}$, separated, and stored at $-20^{\circ} \mathrm{C}$ until assayed. Glucose concentrations were measured using a glucose oxidase method (Yellow Springs Instruments). Plasma insulin was measured using a chemiluminescence assay (Access Assay). C-peptide was measured using a 2-site immunoenzymatic sandwich assay (Roche Diagnostics) in accordance with the manufacturer's instructions. 
Table 2. Pulse and subject characteristics in subjects grouped by glucose tolerance status.

\begin{tabular}{|c|c|c|c|}
\hline & NGT & IGT & $P$ value \\
\hline$n(M / F)$ & $17(5 / 12)$ & $12(5 / 7)$ & $0.06^{A}$ \\
\hline Age (years) & $47 \pm 3$ & $45 \pm 3$ & 0.73 \\
\hline Lean body mass (Kg) & $47.5 \pm 3.1$ & $49.3 \pm 2.4$ & 0.67 \\
\hline BMI $\left(\mathrm{kg} / \mathrm{m}^{2}\right)$ & $27.5 \pm 1.1$ & $28.3 \pm 1.6$ & 0.63 \\
\hline \multicolumn{4}{|c|}{ Screening OGTT data } \\
\hline DI (1 × 10-14 $\mathrm{dL} / \mathrm{kg} / \mathrm{min} / \mathrm{pmol})$ & $1872 \pm 302$ & $620 \pm 122$ & $<0.01$ \\
\hline \multicolumn{4}{|c|}{ Experimental data - fasting phase } \\
\hline Basal secretion (pmol/min) & $121 \pm 8$ & $161 \pm 17$ & 0.07 \\
\hline Amplitude (pmol/min) & $55 \pm 6$ & $65 \pm 9$ & 0.39 \\
\hline Pulse interval (min) & $5.6 \pm 0.5$ & $5.7 \pm 0.5$ & 0.41 \\
\hline Amplitude (pmol/min) & $157 \pm 14$ & $198 \pm 29$ & 0.35 \\
\hline Pulse interval (min) & $5.3 \pm 0.3$ & $4.8 \pm 0.3$ & 0.21 \\
\hline ApEn & $1.20 \pm 0.03$ & $1.15 \pm 0.03$ & 0.25 \\
\hline Frequency dispersion index & $0.40 \pm 0.01$ & $0.43 \pm 0.02$ & 0.34 \\
\hline
\end{tabular}

Assessment of pulsatile insulin secretion by stochastic nonparametric deconvolution and individualized C-peptide kinetics. Deconvolution was utilized to determine the insulin secretion rate over time, or ISR(t), derived from peripheral C-peptide concentrations after assessment of the coefficient of variation (CV) of our C-peptide assay, as previously described (24). To reconstruct the ISR signal, we relied on nonparametric deconvolution (49), which does not require any a priori assumption of the unknown input signal. However, critical to accurate estimation of secretion is knowledge of hormone kinetics. This was directly calculated from experimental data of C-peptide decay in each individual using a Maximum a posteriori estimation $(25,50)$, with a 2-compartment model under the assumption that the C-peptide kinetic is linear (51).

Assessment of insulin secretion pulsatility and pulse characteristics by temporal and frequency analysis. Once the pulsatile ISR(t) was reconstructed, we analyzed the euglycemic and hyperglycemic portion of the signal separately. In each portion, we first calculated the average (basal) ISR during fasting and hyperglycemia (ISR fast and ISR $_{b}$ hyper, respectively) and the above-basal ISR (ISR ${ }_{a b}^{\text {fast }}$ and ISR $_{a b}$ hyper , respectively). ISR $_{a b}$ for each phase of the experiment was taken as $\mathrm{ISR}_{\text {total }}-\mathrm{ISR}_{\mathrm{b}}$. The SD $\left(\mathrm{ISR}_{\mathrm{SD}}\right)$ of $_{\mathrm{ISR}}$ in each phase of the experiment was used as a measure of pulse amplitude. The reconstructed ISR(t) for each individual is shown in Supplemental Figures 1 and 2.

We then calculated the ApEn for the insulin secretion rates (31). ApEn is a validated measure of the regularity and complexity of a temporal series that has been used previously to measure disorderliness in pulsatile insulin secretion $(21,52)$.We subsequently used the FFT algorithm implemented in MATLAB R2017b to obtain the signal power spectra and estimate the main pulse interval. The resulting periodogram for each individual is shown in Supplemental Figures 3-6. The main pulse interval was calculated as the inverse pulse period of the fastest harmonic in the spectrum that had an amplitude of $\geq 40 \%$ of the peak amplitude. This threshold was chosen empirically, under the assumption that spectrum peaks, with amplitude less than $40 \%$ of peak amplitude, do not contribute significantly to the main pulsatile pattern observed peripherally. The pulse interval thus calculated represents an estimate of the average time interval between 2 pulses.

In order to provide a more rigorous measurement of pulsatility and better characterize the contribution of different pulse frequencies to overall secretion, we first applied a high-pass filter with a cut-off frequency 
of $8.3 \times 10^{-4} \mathrm{~Hz}$ in order to remove the effect of slow frequencies (those with a pulse interval longer than 20 minutes). This enabled us to focus on pulses with a frequency that contributes to minute-to-minute pulsatility (0-20 minutes, as previously reported; ref. 53). To quantify the variability of the frequencies, we used a FDI. This index (between 0 and 1) increases as the signal bandwidth increases, and it is defined by:

$$
F D I=\sqrt{1-\frac{\mu_{1}^{2}}{\mu_{0} \mu_{2}}} \text { where } \mu_{\mathrm{n}} \text { is the }-n \text {th statistical moment of the } \operatorname{spectrum}(30) \text {. }
$$

Statistics. Previously, Meier et al. measured pulsatile insulin secretion in 6 healthy adults (16). In that experiment, pulses measured in the peripheral vein occurred with a periodicity of $4.20 \pm 0.24 \mathrm{~min} /$ pulse and an amplitude of $59 \pm 20 \mathrm{pmol} / \mathrm{L}$ (mean $\pm \mathrm{SD}$ ). Assuming similar variation, with 13 subjects in each genotype group, we would have $80 \%$ power to detect a $6 \%$ and $39 \%$ change in mean pulse frequency and amplitude, respectively, at an $\alpha$ of 0.05 .

Our data and results are presented as mean \pm SEM. Between-group comparisons were performed using an unpaired, 2-tailed Student $t$ test for normally distributed variables; conversely, when samples were not normally distributed, a 2-tailed Wilcoxon test was used. A paired, 2-way student $t$ test (parametric) or Wilcoxon matched-pairs signed rank test (nonparametric) was used to examine within-group changes (fasting vs. hyperglycemia). The $\chi^{2}$ test was used to examine between-group differences in sex and glucose-tolerance status. $P<0.05$ was considered statistically significant. Analysis was performed in Prism 5 (GraphPad Software, San Diego, CA).

Study approval. After approval by the Mayo Clinic IRB, 29 nondiabetic subjects, recruited from among subjects who had previously participated in a series of published experiments $(6,25,54)$, provided informed, written consent to participate in this study. All were recruited on the basis of genotype at rs7903146, as outlined previously (6).

\section{Author contributions}

MCL and CDM developed the method, analyzed the data, and reviewed/edited manuscript. RTV researched data and ran the studies. RAR, AM, and GDN contributed to the discussion and reviewed/ edited manuscript. CC developed the method and reviewed/edited manuscript. AV designed the study, oversaw its conduct, researched data, contributed to the discussion, and wrote the manuscript. JCA placed the hepatic vein catheter in each subject, contributed to the discussion, and reviewed and edited the manuscript. AV is the guarantor of this work and, as such, had full access to all the data in the study and takes responsibility for the integrity of the data and the accuracy of the data analysis.

\section{Acknowledgments}

This study was supported by US NIH (DK78646, DK116231), University of Padova Research Grant CPDA145405 and Mayo Clinic General Clinical Research Center (UL1 TR000135).

Address correspondence to: Adrian Vella, Endocrine Research Unit, Mayo Clinic College of Medicine, 200 First St. SW, 5-194 Joseph, Rochester, Minnesota 55905, USA. Phone: 507.255.6515; Email: vella.adrian@mayo.edu.

1. Mahajan A, et al. Fine-mapping type 2 diabetes loci to single-variant resolution using high-density imputation and islet-specific epigenome maps. Nat Genet. 2018;50(11):1505-1513.

2. Khera AV, et al. Genome-wide polygenic scores for common diseases identify individuals with risk equivalent to monogenic mutations. Nat Genet. 2018;50(9):1219-1224.

3. Talmud PJ, et al. Sixty-five common genetic variants and prediction of type 2 diabetes. Diabetes. 2015;64(5):1830-1840.

4. Morris AP, et al. Large-scale association analysis provides insights into the genetic architecture and pathophysiology of type 2 diabetes. Nat Genet. 2012;44(9):981-990.

5. Loos RJ, et al. TCF7L2 polymorphisms modulate proinsulin levels and beta-cell function in a British Europid population. Diabetes. 2007;56(7):1943-1947.

6. Shah M, et al. TCF7L2 Genotype and $\alpha$-Cell Function in Humans Without Diabetes. Diabetes. 2016;65(2):371-380.

7. Shah P, Vella A, Basu A, Basu R, Schwenk WF, Rizza RA. Lack of suppression of glucagon contributes to postprandial hyperglycemia in subjects with type 2 diabetes mellitus. J Clin Endocrinol Metab. 2000;85(11):4053-4059.

8. Bertram R, Satin LS, Sherman AS. Closing in on the Mechanisms of Pulsatile Insulin Secretion. Diabetes. 2018;67(3):351-359.

9. Pedersen MG, Bertram R, Sherman A. Intra- and inter-islet synchronization of metabolically driven insulin secretion. Biophys $J$. 2005;89(1):107-119. 
10. Pedersen MG, Mosekilde E, Polonsky KS, Luciani DS. Complex patterns of metabolic and $\mathrm{Ca}^{2}+$ entrainment in pancreatic islets by oscillatory glucose. Biophys J. 2013;105(1):29-39.

11. Fendler B, Zhang M, Satin L, Bertram R. Synchronization of pancreatic islet oscillations by intrapancreatic ganglia: a modeling study. Biophys J. 2009;97(3):722-729.

12. Benninger RK, Piston DW. Cellular communication and heterogeneity in pancreatic islet insulin secretion dynamics. Trends Endocrinol Metab. 2014;25(8):399-406.

13. Duckworth WC, Bennett RG, Hamel FG. Insulin degradation: progress and potential. Endocr Rev. 1998;19(5):608-624.

14. Pørksen N, et al. Human insulin release processes measured by intraportal sampling. Am J Physiol Endocrinol Metab. 2002;282(3):E695-E702.

15. Sathananthan A, et al. A concerted decline in insulin secretion and action occurs across the spectrum of fasting and postchallenge glucose concentrations. Clin Endocrinol (Oxf). 2012;76(2):212-219.

16. Meier JJ, Veldhuis JD, Butler PC. Pulsatile insulin secretion dictates systemic insulin delivery by regulating hepatic insulin extraction in humans. Diabetes. 2005;54(6):1649-1656.

17. Pørksen N. Early changes in beta-cell function and insulin pulsatility as predictors for type 2 diabetes. Diabetes Nutr Metab. 2002;15(6 Suppl):9-14.

18. O'Rahilly S, Turner RC, Matthews DR. Impaired pulsatile secretion of insulin in relatives of patients with non-insulin-dependent diabetes. N Engl J Med. 1988;318(19):1225-1230.

19. Goodner CJ, Koerker DJ, Weigle DS, McCulloch DK. Decreased insulin- and glucagon-pulse amplitude accompanying betacell deficiency induced by streptozocin in baboons. Diabetes. 1989;38(7):925-931.

20. Pørksen NK, Munn SR, Steers JL, Schmitz O, Veldhuis JD, Butler PC. Mechanisms of sulfonylurea's stimulation of insulin secretion in vivo: selective amplification of insulin secretory burst mass. Diabetes. 1996;45(12):1792-1797.

21. Pørksen N, et al. Glucagon-like peptide 1 increases mass but not frequency or orderliness of pulsatile insulin secretion. Diabetes. 1998;47(1):45-49.

22. Biden TJ, Boslem E, Chu KY, Sue N. Lipotoxic endoplasmic reticulum stress, $\beta$ cell failure, and type 2 diabetes mellitus. Trends Endocrinol Metab. 2014;25(8):389-398.

23. Florez JC, et al. TCF7L2 polymorphisms and progression to diabetes in the Diabetes Prevention Program. $N$ Engl J Med. 2006;355(3):241-250.

24. Laurenti MC, et al. Assessment of pulsatile insulin secretion derived from peripheral plasma C-peptide concentrations by nonparametric stochastic deconvolution. Am J Physiol Endocrinol Metab. 2019;316(5):E687-E694.

25. Varghese RT, et al. Performance of individually measured vs population-based C-peptide kinetics to assess $\beta$-cell function in the presence and absence of acute insulin resistance. Diabetes Obes Metab. 2018;20(3):549-555.

26. Meneilly GS, Ryan AS, Veldhuis JD, Elahi D. Increased disorderliness of basal insulin release, attenuated insulin secretory burst mass, and reduced ultradian rhythmicity of insulin secretion in older individuals. J Clin Endocrinol Metab. 1997;82(12):4088-4093

27. Ahmed S, Bennett ST, Huxtable SJ, Todd JA, Matthews DR, Gough SC. INS VNTR allelic variation and dynamic insulin secretion in healthy adult non-diabetic Caucasian subjects. Diabet Med. 1999;16(11):910-917.

28. Dunger DB, Matthews DR, Edge JA, Jones J, Preece MA. Evidence for temporal coupling of growth hormone, prolactin, LH and FSH pulsatility overnight during normal puberty. J Endocrinol. 1991;130(1):141-149.

29. Matthews JN, Altman DG, Campbell MJ, Royston P. Analysis of serial measurements in medical research. $B M J$. 1990;300(6719):230-235.

30. Prieto TE, Myklebust JB, Hoffmann RG, Lovett EG, Myklebust BM. Measures of postural steadiness: differences between healthy young and elderly adults. IEEE Trans Biomed Eng. 1996;43(9):956-966.

31. Pincus SM. Approximate entropy as a measure of system complexity. Proc Natl Acad Sci USA. 1991;88(6):2297-2301.

32. Humphries SE, et al. Common variants in the TCF7L2 gene and predisposition to type 2 diabetes in UK European Whites, Indian Asians and Afro-Caribbean men and women. J Mol Med. 2006;84(12):1005-1014.

33. Lyssenko V, et al. Mechanisms by which common variants in the TCF7L2 gene increase risk of type 2 diabetes. J Clin Invest. 2007;117(8):2155-2163.

34. Munoz J, et al. Polymorphism in the transcription factor 7-like 2 (TCF7L2) gene is associated with reduced insulin secretion in nondiabetic women. Diabetes. 2006;55(12):3630-3634.

35. Xia Q, et al. The type 2 diabetes presumed causal variant within TCF7L2 resides in an element that controls the expression of ACSL5. Diabetologia. 2016;59(11):2360-2368.

36. van de Bunt M. An alternative effector gene at the type 2 diabetes-associated TCF7L2 locus? Diabetologia. 2016;59(11):2292-2294

37. Loder MK, da Silva Xavier G, McDonald A, Rutter GA. TCF7L2 controls insulin gene expression and insulin secretion in mature pancreatic beta-cells. Biochem Soc Trans. 2008;36(Pt 3):357-359.

38. da Silva Xavier G, et al. TCF7L2 regulates late events in insulin secretion from pancreatic islet beta-cells. Diabetes. 2009;58(4):894-905.

39. Rosengren $\mathrm{AH}$, et al. Reduced insulin exocytosis in human pancreatic $\beta$-cells with gene variants linked to type 2 diabetes. Diabetes. 2012;61(7):1726-1733.

40. Mitchell RK, et al. Selective disruption of Tcf712 in the pancreatic $\beta$ cell impairs secretory function and lowers $\beta$ cell mass. Hum Mol Genet. 2015;24(5):1390-1399.

41. Sorrenson B, et al. A Critical Role for $\beta$-Catenin in Modulating Levels of Insulin Secretion from $\beta$-Cells by Regulating Actin Cytoskeleton and Insulin Vesicle Localization. J Biol Chem. 2016;291(50):25888-25900.

42. Juhl CB, Schmitz O, Pincus S, Holst JJ, Veldhuis J, Pørksen N. Short-term treatment with GLP-1 increases pulsatile insulin secretion in Type II diabetes with no effect on orderliness. Diabetologia. 2000;43(5):583-588.

43. Laedtke T, et al. Overnight inhibition of insulin secretion restores pulsatility and proinsulin/insulin ratio in type 2 diabetes. $A m$ J Physiol Endocrinol Metab. 2000;279(3):E520-E528.

44. Cobelli C, Dalla Man C, Toffolo G, Basu R, Vella A, Rizza R. The oral minimal model method. Diabetes. 2014;63(4):1203-1213.

45. Bock G, et al. Pathogenesis of pre-diabetes: mechanisms of fasting and postprandial hyperglycemia in people with impaired fasting glucose and/or impaired glucose tolerance. Diabetes. 2006;55(12):3536-3549. 
46. Shankar SS, et al. Standardized Mixed-Meal Tolerance and Arginine Stimulation Tests Provide Reproducible and Complementary Measures of $\beta$-Cell Function: Results From the Foundation for the National Institutes of Health Biomarkers Consortium Investigative Series. Diabetes Care. 2016;39(9):1602-1613.

47. Dalla Man C, et al. Two-hour seven-sample oral glucose tolerance test and meal protocol: minimal model assessment of betacell responsivity and insulin sensitivity in nondiabetic individuals. Diabetes. 2005;54(11):3265-3273.

48. Service GJ, Thompson GB, Service FJ, Andrews JC, Collazo-Clavell ML, Lloyd RV. Hyperinsulinemic hypoglycemia with nesidioblastosis after gastric-bypass surgery. NEngl J Med. 2005;353(3):249-254.

49. De Nicolao G, Sparacino G, Cobelli C. Nonparametric input estimation in physiological systems problems, methods, and case studies. Automatica. 1997;33(5):851-870.

50. Sparacino G, Tombolato C, Cobelli C. Maximum-likelihood versus maximum a posteriori parameter estimation of physiological system models: the C-peptide impulse response case study. IEEE Trans Biomed Eng. 2000;47(6):801-811.

51. Eaton RP, Allen RC, Schade DS, Erickson KM, Standefer J. Prehepatic insulin production in man: kinetic analysis using periph eral connecting peptide behavior. J Clin Endocrinol Metab. 1980;51(3):520-528.

52. Hollingdal M, et al. Failure of physiological plasma glucose excursions to entrain high-frequency pulsatile insulin secretion in type 2 diabetes. Diabetes. 2000;49(8):1334-1340.

53. Pørksen N, Hollingdal M, Juhl C, Butler P, Veldhuis JD, Schmitz O. Pulsatile insulin secretion: detection, regulation, and role in diabetes. Diabetes. 2002;51 Supp1 1:S245-S254.

54. Varghese RT, et al. Diabetes-Associated Variation in TCF7L2 Is Not Associated With Hepatic or Extrahepatic Insulin Resistance. Diabetes. 2016;65(4):887-892. 